\title{
Primary Choroidal Lymphoma Diagnosed with 27-Gauge Pars Plana Vitrectomy Choroidal Biopsy
}

\author{
Andrew W. Kam ${ }^{a, b}$ Justin Galvin ${ }^{c}$ Svetlana Cherepanoff ${ }^{\mathrm{d}, \mathrm{e}}$ \\ A. Andrew Miller ${ }^{f, g} \quad$ Adrian T. Fung ${ }^{\text {h-j }}$ \\ aRoyal North Shore Hospital, Sydney, NSW, Australia; ${ }^{b}$ The University of Sydney,

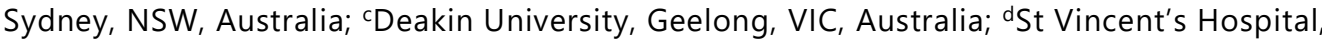

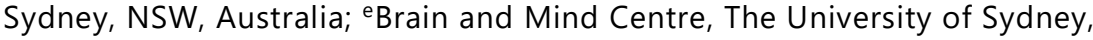 \\ Sydney, NSW, Australia; flllawarra Cancer Care Centre, Wollongong Hospital, \\ Wollongong, NSW, Australia; ${ }^{C}$ Centre for Oncology Informatics, University of Wollongong, \\ Gwynneville, NSW, Australia; hSave Sight Institute, The University of Sydney, \\ Sydney, NSW, Australia; 'Westmead Hospital, Sydney, NSW, Australia; ${ }^{j}$ Faculty of Medicine \\ and Health Sciences, Macquarie University, Macquarie Park, NSW, Australia
}

\section{Keywords}

Choroidal lymphoma $\cdot 27$-gauge vitrectomy $\cdot$ Extranodal marginal zone lymphoma

\begin{abstract}
Background: Currently, transvitreal fine-needle aspiration biopsy is the most widely used tissue biopsy technique in cases of suspected intraocular lymphoma due to its relative simplicity and low trauma. The small sample produced, however, may be inadequate for diagnostic and prognostic analyses due to mechanical artefacts, insufficient material, or sampling errors. Small case series have demonstrated choroidal biopsy via vitrectomy to be safe and effective. With smaller-gauge vitrectomy instruments, visual recovery is rapid, and post-operative inflammation and conjunctival scarring is minimised. Furthermore, smaller-gauge instrumentation does not appear to affect the diagnostic yield of biopsies for intraocular lymphoma in vitro. We report a case of primary choroidal lymphoma successfully diagnosed with 27-gauge pars plana vitrectomy choroidal biopsy. Case Presentation: A 72-year-old female presented with a 6month history of painless blurred vision in her right eye. Fundus examination revealed a large pale choroidal mass centred on the posterior pole with overlying exudative retinal detachment.
\end{abstract}




\section{Case Reports in Ophthalmology}

Enhanced depth imaging optical coherence tomography revealed a markedly thickened choroid with an undulating appearance. B-scan ultrasonography demonstrated diffuse, smooth thickening of the choroid, and retrobulbar extrascleral hypoechoic nodules. A 27-gauge pars plana vitrectomy was performed and choroidal biopsy taken. Histopathologic, immunohistochemical, and flow cytometry studies confirmed a diagnosis of extranodal marginal zone Bcell lymphoma. Systemic workup found no evidence of systemic lymphoma. As such, the patient was diagnosed with primary choroidal lymphoma. She underwent intensity-modulated external beam radiotherapy with subsequent resolution of disease. Conclusions: Primary choroidal lymphoma can be safely and effectively diagnosed via 27 -gauge vitrectomy choroidal biopsy.

\section{Introduction}

Primary choroidal lymphoma is a rare form of intraocular malignancy, accounting for few case reports and case series in the literature [1,2]. It is defined as primary if there is an absence of systemic lymphoma at the time of ocular presentation [2]. Primary choroidal lymphoma typically presents in patients 60 years and older with monocular, slowly progressive blurring of vision. However, up to $20 \%$ of cases are asymptomatic [1, 2]. Typically, histopathological analysis reveals extranodal marginal zone B-cell lymphoma (EMZL) [2-4].

Diagnosis of choroidal lymphoma can be challenging. The differential diagnosis is wide and includes amelanotic choroidal melanoma, choroidal metastasis, choroidal haemangioma, sarcoidosis, syphilis, tuberculosis, and other granulomatous choroidopathies [4]. As such, patients frequently remain undiagnosed for extended periods before a correct diagnosis is made and proper treatment is initiated [1,2]. A tissue biopsy is required for diagnosis and therapy decisions. In this case report, we illustrate the utility of 27 -gauge pars plana vitrectomy with choroidal biopsy for the diagnosis of primary choroidal lymphoma.

\section{Case Presentation}

A 72-year-old Caucasian female with an ocular history of right amblyopia and baseline best-corrected visual acuity (BCVA) of 20/40 presented with a 6-month history of painless blurred vision in her right eye. She had no other significant medical or ophthalmic history. On examination, BCVA was hand movements in the right eye, and 20/20 in the left eye. Fundus examination of the right eye revealed a large pale choroidal mass centred at the posterior pole with overlying exudative retinal detachment (Fig. 1a). The left eye was normal.

Fluorescein angiography (Zeiss VISUCAM ${ }^{\circledR} 500$, Carl Zeiss Meditec AG, Jena, Germany) demonstrated hyperfluorescence focussed over the choroidal lesion, but on indocyanine green angiography, the lesion was hypofluorescent (Fig. 1b, c). Enhanced-depth imaging optical coherence tomography (EDI-OCT, Heidelberg Spectralis HRA camera, Heidelberg Engineering, Heidelberg, Germany) showed subretinal fluid, subretinal hyperreflective material, and cystic degeneration of the retina (Fig. 1d). The choroid was markedly thickened with an undulating appearance and low hyperreflective homogenicity. B-scan ultrasonography (Eye Cubed $^{\mathrm{TM}}$, Ellex, Mawson Lakes, Australia) revealed a thickened, hypoechoic choroid with retrobulbar extrascleral hypoechoic nodules (Fig. 1e). Complete blood count, serum protein electrophoresis, angiotensin-converting enzyme levels, Quantiferon gold testing, and syphilis serology revealed no abnormalities. 


\section{Case Reports in Ophthalmology}

The clinical examination and investigations were suggestive of a choroidal lymphoproliferative process. As such, a 27-gauge pars plana vitrectomy was performed and a choroidal biopsy taken in the temporal part of the lesion with a 27-gauge cutter. Vitrectomy-assisted choroidal biopsy, including 27 -gauge, had been well described for choroidal melanoma [5-7]. It had not to our knowledge, however, been described for choroidal lymphoma. Minimal vitrectomy was performed (just sufficient to safely approach the choroid) and the hyaloid was not elevated (to reduce the risk of tears/detachment). The intraocular pressure was raised to $60 \mathrm{~mm} \mathrm{Hg}$ at the time of biopsy to reduced haemorrhage, and slowly lowered post-biopsy. The cut rate was reduced to 500 cuts per minute with high suction $(600 \mathrm{~mm} \mathrm{Hg})$. A 5-mL syringe was directly attached to the aspiration line and manually drawn by a surgical assistant at the time of biopsy. The vitrectomy cutter was gently rotated to maximise yield of tissue. No laser retinopexy to the biopsy site was required. A partial air tamponade was used at the end of the vitrectomy to assist sealing the sclerotomies and to check for leakage, as per the surgeon's routine practice. A second biopsy was also taken from an area of yellow thickened superior conjunctiva that was identified as potentially abnormal at the time of surgery.

The choroidal biopsy showed a monomorphous population of CD20, BCL-2 positive, CD10, cyclin D1, CD23, and CD5 small lymphocytes (Fig. 2a, c). On flow cytometry, they were found to be an abnormal B-cell population that was CD19, CD20, and kappa light chain positive, and CD5, CD10, CD11c, CD22, CD23, and CD200 negative. The conjunctival biopsy showed similar results to the choroidal biopsy with an atypical infiltrate of small lymphoid B cells within the substantia propria (Fig. 2b) and an identical immunophenotype on flow cytometry. Based on the histopathologic, immunohistochemical, and flow cytometry studies, a diagnosis of EMZL was made.

In view of the histopathological findings, workup for systemic involvement was performed with magnetic resonance imaging (MRI) of the brain, orbits, and spine, and computed tomography (CT) of the chest, abdomen, and pelvis. MRI demonstrated diffuse thickening and enhancement of the right choroid and an $8 \times 7 \mathrm{~mm}$ soft tissue nodule posterior to the globe in the intraconal space lying between the optic nerve and lateral rectus. There was no other evidence of intracranial or spinal lymphoma. CT showed mild mediastinal and bilateral hilar lymphadenopathy. Subsequent fine needle aspiration of the enlarged subcarinal lymph nodes showed granulomatous lymphadenitis suggestive of sarcoidosis. There was no evidence of metastatic malignancy.

As the disease was localised to the right orbit, the patient underwent intensity-modulated external beam radiation therapy. The clinical target volume included the contents of the entire right orbit with a movement envelope of $2 \mathrm{~mm}$ added to produce a planning target volume which initially received 4 Gy in 2 fractions. A small $7 \times 4 \mathrm{~mm}$ persistent enhancing nodule was noted on the 5-week post-therapy progress MRI. Given the lack of complete response, a further $24 \mathrm{~Gy}$ in 12 fractions were delivered to the previous planning target volume. Two months following the second course of radiotherapy, progress MRI showed shrinking of the nodule to $5 \times 3 \mathrm{~mm}$ with reduction in contrast enhancement. Nine months following the second course of radiotherapy, progress MRI showed further shrinking of the nodule to $3 \times 3 \mathrm{~mm}$. While unlikely that this tissue represents residual disease, it continues to be followed up with regular progress scans.

Three months following radiotherapy (7 months post-operative), visual acuity in the right eye remained poor at count fingers. Fundus examination of the right eye revealed a residual area of pallor over the previous site of the choroidal lesion (Fig. 3a). On EDI-OCT, there was resolution of the subretinal fluid and hyperreflective material with thinning of the choroid. There was attenuation of the retinal pigment epithelium and the other outer retina including 
the ellipsoid line (Fig. 3b). B-scan ultrasonography revealed resolution of the choroidal thickening and extrascleral nodules (Fig. 3c).

\section{Discussion}

Primary intraocular lymphomas arise from different parts of the eye and may have a wide range of clinical manifestations, masquerading as many other diseases [4]. Primary uveal lymphoma, the most common of which is primary choroidal lymphoma, differs in a number of respects to other types of primary intraocular lymphoma, the most common of which are primary vitreoretinal lymphomas $[4,8]$. For example, while vitreoretinal lymphomas are typically high-grade malignancies usually associated with central nervous system disease, primary choroidal lymphomas are typically low-grade and clinically indolent, with central nervous system spread being relatively rare $[4,8]$.

In cases of suspected intraocular lymphoma, tissue biopsies are useful for excluding inflammatory diseases and for subtyping of neoplastic lesions. Such information is essential for planning treatment. Currently, transvitreal fine-needle aspiration biopsy is the most widely used biopsy technique due to its relative simplicity and low trauma $[2,9,10]$. Needle gauges used are typically 25-gauge or 27-gauge [11]. The small sample produced, however, may be inadequate for diagnostic and prognostic analyses due to mechanical artefacts, insufficient material, or sampling errors $[9,10]$. The diagnostic yield for fine-needle aspiration biopsy has been shown to be inadequate in $5-12 \%$ of cases [12].

Small case series have demonstrated choroidal biopsy via vitrectomy to be safe, effective, and relatively quick to perform $[5,10,13]$. With smaller-gauge vitrectomy instruments, visual recovery is rapid, and post-operative inflammation and conjunctival scarring is minimised [14]. Furthermore, smaller-gauge instrumentation does not appear to affect the diagnostic yield of biopsies for intraocular lymphoma in vitro $[15,16]$. While we are not aware of any cases of 27-gauge vitrectomy-assisted choroidal biopsy for choroidal lymphoma in vivo, 25gauge to 27-gauge instrumentation has demonstrated diagnostic yields between $88.9-100 \%$ for choroidal melanoma [5]. Our case demonstrates that choroidal biopsy using 27-gauge instrumentation is feasible and yields adequate specimens for histopathological, immunohistochemical, and flow cytometry studies. The size of the vitrector cutter is no larger than that of a fine needle but allows for direct (rather than inverted) visualisation and for a larger specimen to be obtained.

Choroidal lymphoma may be associated with anterior or posterior epibulbar extension of the lymphoid proliferation [2]. In the largest case study of choroidal lymphoma to date by Mashayekhi et al. [2], 50\% of patients who underwent biopsy to confirm the diagnosis underwent anterior epibulbar biopsy. In our case, the abnormal area of superior bulbar conjunctiva, which was later found to be EMZL, was only identified at the time of surgery. As such, careful examination of the conjunctiva may have precluded the need for choroidal biopsy.

Optimal treatment regimens for choroidal lymphoma have not yet been established due to the small number of patients with the disease and the lack of studies with long-term followup [1]. In recent times, however, external beam radiation therapy has been the most frequently used modality in many institutions for localised disease [1, 17]. Chemotherapy and immunotherapy have been reserved for patients with multifocal or systemic disease $[1,17]$. Indeed, external beam radiation therapy appears to be sufficient to successfully manage localised choroidal lymphoma. This was shown in a retrospective review of 25 eyes with choroidal lymphoma treated with external beam radiation therapy alone where complete regression 
Kam et al.: Primary Choroidal Lymphoma Diagnosed with 27-Gauge Pars Plana Vitrectomy Choroidal Biopsy

was achieved in 18 eyes (72\%) and partial regression in 6 eyes (24\%) at a mean follow-up of 58 months with doses averaging 2,867 cGy (median 3,000 cGy, range 1,200-3,600 cGy) (note: one patient had systemic rituximab initially but went on to have external beam radiation therapy because of lack of improvement) [17].

A number of imaging features can suggest the diagnosis of choroidal lymphoma. On EDIOCT, there is often a hyperreflective signal at the retinal pigment epithelium-Bruch membrane junction, with the topography of the choroid varying from smooth and flat in early disease to an undulating, "wave-like," or "seasick" appearance in advanced disease [18, 19]. This differs from most choroidal tumours, which demonstrate a smooth anterior dome or plateau-shaped surface $[18,19]$. On B-scan ultrasonography, choroidal lymphoma typically has the appearance of a smooth-surfaced, hypoechoic, cresenteric thickening of the choroid. Hypoechoic areas of posterior epibulbar extension may also be present $[1,2]$.

As choroidal lymphoma may be the first presentation of systemic lymphoma, a thorough systemic evaluation should be performed in all patients at initial diagnosis [2, 3]. Subsequent late development of systemic lymphoma occurs very rarely [2,3], which should be borne in mind during follow-up. Furthermore, in the setting of systemic lymphadenopathy and suspicious choroidal lesions, biopsy of extraocular sites alone is insufficient in ruling out primary choroidal lymphoma. This was demonstrated in our case, as biopsy of the systemic lesions alone would have suggested a diagnosis of sarcoidosis and led to a very different treatment pathway. If a choroidal biopsy had not been undertaken, the diagnosis of lymphoma would have been missed.

In summary, primary choroidal lymphoma can be safely and effectively diagnosed via 27gauge vitrectomy choroidal biopsy.

\section{Statement of Ethics}

This research was conducted in accordance with the World Medical Association Declaration of Helsinki. The patient provided written informed consent for the creation and publication of this report.

\section{Disclosure Statement}

The authors have no conflicts of interest to declare.

\section{Funding Sources}

No funding or grant support.

\section{Author Contributions}

A.W.K., J.G., and A.T.F. were major contributors in writing the manuscript. S.C. performed and interpreted the histopathological analysis of the biopsy samples. A.A.M. planned and executed the radiotherapy regimens. A.T.F. conceived, designed, and oversaw the study and performed the workup and surgical procedure. All authors have edited, read, and approved the final manuscript. 


\section{Case Reports in Ophthalmology}

\section{References}

1 Aronow ME, Portell CA, Sweetenham JW, Singh AD. Uveal lymphoma: clinical features, diagnostic studies, treatment selection, and outcomes. Ophthalmology. 2014 Jan;121(1):334-41.

2 Mashayekhi A, Shukla SY, Shields JA, Shields CL. Choroidal lymphoma: clinical features and association with systemic lymphoma. Ophthalmology. 2014 Jan;121(1):342-51.

3 Coupland SE, Foss HD, Hidayat AA, Cockerham GC, Hummel M, Stein H. Extranodal marginal zone B cell lymphomas of the uvea: an analysis of 13 cases. J Pathol. 2002 Jul;197(3):333-40.

4 Coupland SE, Damato B. Understanding intraocular lymphomas. Clin Exp Ophthalmol. 2008 Aug;36(6):56478.

5 Grewal DS, Cummings TJ, Mruthyunjaya P. Outcomes of 27-Gauge Vitrectomy-Assisted Choroidal and Subretinal Biopsy. Ophthalmic Surg Lasers Imaging Retina. 2017 May;48(5):406-15.

6 Finn AP, Materin MA, Mruthyunjaya P. CHOROIDAL TUMOR BIOPSY: A Review of the Current State and a Glance Into Future Techniques. Retina. 2018 Sep;38 Suppl 1:S79-87.

7 Reddy DM, Mason LB, Mason JO 3rd, Crosson JN, Yunker JJ. Vitrectomy and Vitrector Port Needle Biopsy of Choroidal Melanoma for Gene Expression Profile Testing Immediately before Brachytherapy. Ophthalmology. 2017 Sep;124(9):1377-82.

8 Coupland SE, Heimann H, Bechrakis NE. Primary intraocular lymphoma: a review of the clinical, histopathological and molecular biological features. Graefes Arch Clin Exp Ophthalmol. 2004 Nov;242(11):901-13.

9 Coupland SE, Joussen A, Anastassiou G, Stein H. Diagnosis of a primary uveal extranodal marginal zone B-cel lymphoma by chorioretinal biopsy: case report. Graefes Arch Clin Exp Ophthalmol. 2005 May;243(5):482-6.

10 Sen J, Groenewald C, Hiscott PS, Smith PA, Damato BE. Transretinal choroidal tumor biopsy with a 25-gauge vitrector. Ophthalmology. 2006 Jun;113(6):1028-31.

11 McCannel TA. Fine-needle aspiration biopsy in the management of choroidal melanoma. Curr Opin Ophthalmol. 2013 May;24(3):262-6.

12 Singh AD, Biscotti CV. Fine needle aspiration biopsy of ophthalmic tumors. Saudi J Ophthalmol. 2012 Apr;26(2):117-23.

13 Mastropasqua R, Thaung C, Pavesio C, Lightman S, Westcott M, Okhravi N, et al. The Role of Chorioretinal Biopsy in the Diagnosis of Intraocular Lymphoma. Am J Ophthalmol. 2015 Dec;160(6):1127-1132.e1.

14 Khan MA, Kuley A, Riemann CD, Berrocal MH, Lakhanpal RR, Hsu J, et al. Long-Term Visual Outcomes and Safety Profile of 27-Gauge Pars Plana Vitrectomy for Posterior Segment Disease. Ophthalmology. 2018 Mar;125(3):423-31.

15 Fortun JA, Carvounis PE, Albini TA, Holz ER, Chevez-Barrios P. Comparison of Cytologic and Flow Cytometry Features of Vitreous Samples Obtained Using 20- or 25-Gauge Vitrectomy for the Diagnosis of Lymphoma. Invest Ophthalmol Vis Sci. 2007;48(13):2212.

16 Trikha R, Yeung CC, Modjtahedi SP, Telander DG. Evaluation of 25 Gauge and 20 Gauge Vitrectomy on Cell Viability and Diagnostic Yield for B-Cell Lymphoma in Culture Using Flow Cytometry. Invest Ophthalmol Vis Sci. 2009;50(13):5750.

17 Mashayekhi A, Hasanreisoglu M, Shields CL, Shields JA. EXTERNAL BEAM RADIATION FOR CHOROIDAL LYMPHOMA: efficacy and Complications. Retina. 2016 Oct;36(10):2006-12.

18 Shields CL, Arepalli S, Pellegrini M, Mashayekhi A, Shields JA. Choroidal lymphoma shows calm, rippled, or undulating topography on enhanced depth imaging optical coherence tomography in 14 eyes. Retina. 2014 Jul;34(7):1347-53.

19 Arias JD, Kumar N, Fulco EA, Spaide R, Yannuzzi L, Shields JA, et al. The seasick choroid: a finding on enhanced depth imaging spectral-domain optical coherence tomography of choroidal lymphoma. Retin Cases Brief Rep. 2013;7(1):19-22. 


\section{Case Reports in Ophthalmology}
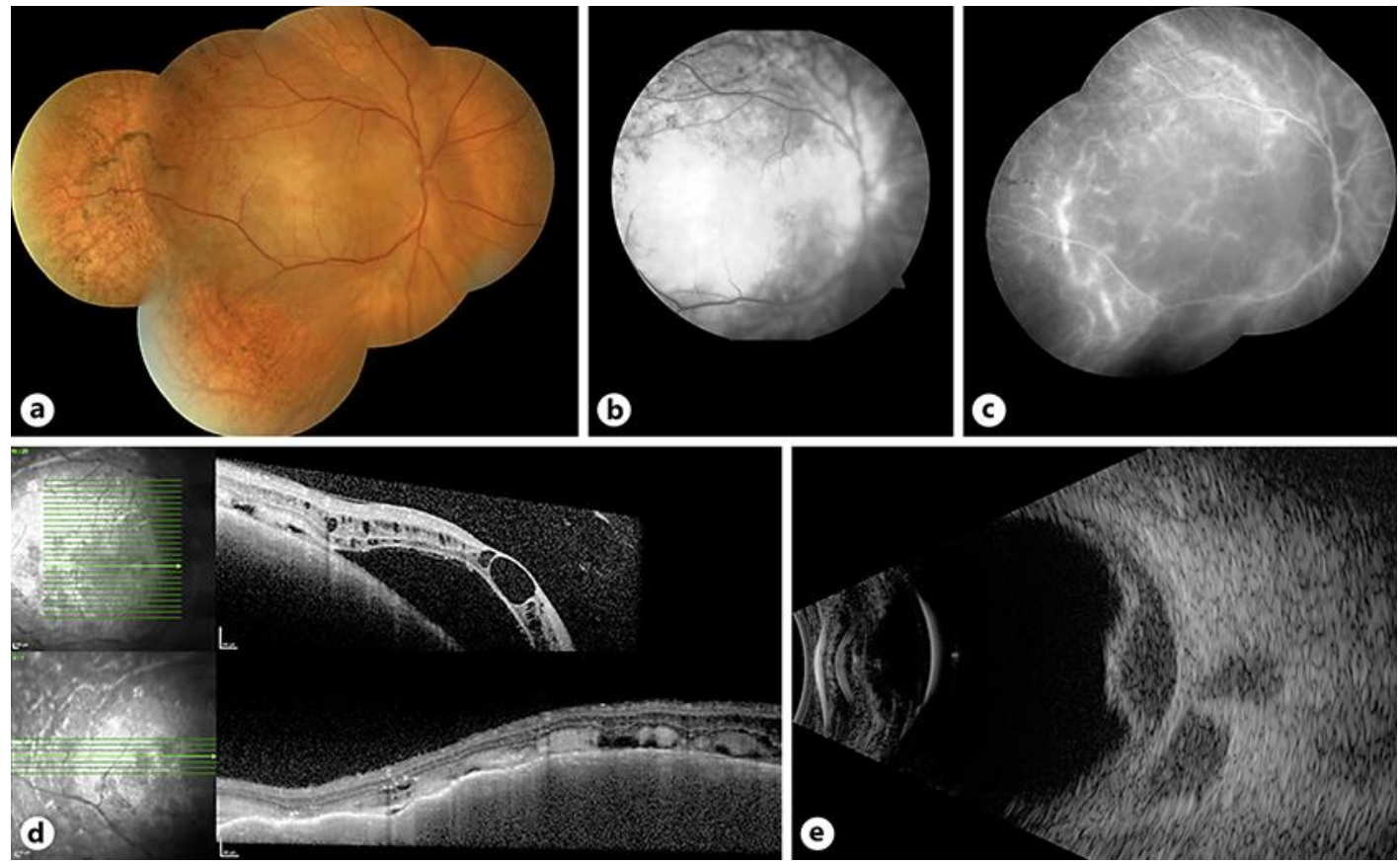

Fig. 1. a Colour fundus photograph of the right eye reveals a large pale choroidal mass centred on the posterior pole with overlying exudative retinal detachment. b Fluorescein angiography taken at 5 min $10 \mathrm{~s}$ demonstrates diffuse hyperfluorescence focussed over the choroidal lesion. c Indocyanine green angiography shows relative hypofluorescence of the posterior pole with large dilated hyperfluorescent choroidal vessels. d Enhanced depth imaging optical coherence tomography scan of the right macula shows sub- and intraretinal fluid at the macula (top) and hyperreflective subretinal material with marked thickening of the underlying hyperreflective choroid through the temporal aspect of the lesion (bottom). e An axial 12 o'clock meridional B-scan ultrasound demonstrates a thickened, hypoechoic choroid. In addition, there are retrobulbar extrascleral hypoechoic nodules. 


\section{Case Reports in Ophthalmology}

\begin{tabular}{l|l}
\hline Case Rep Ophthalmol 2019;10:213-220 \\
\hline DOI: 10.1159/000500238 & $\begin{array}{l}\text { @ 2019 The Author(s). Published by S. Karger AG, Basel } \\
\text { www.karger.com/cop }\end{array}$ \\
\hline
\end{tabular}

Kam et al.: Primary Choroidal Lymphoma Diagnosed with 27-Gauge Pars Plana Vitrectomy Choroidal Biopsy
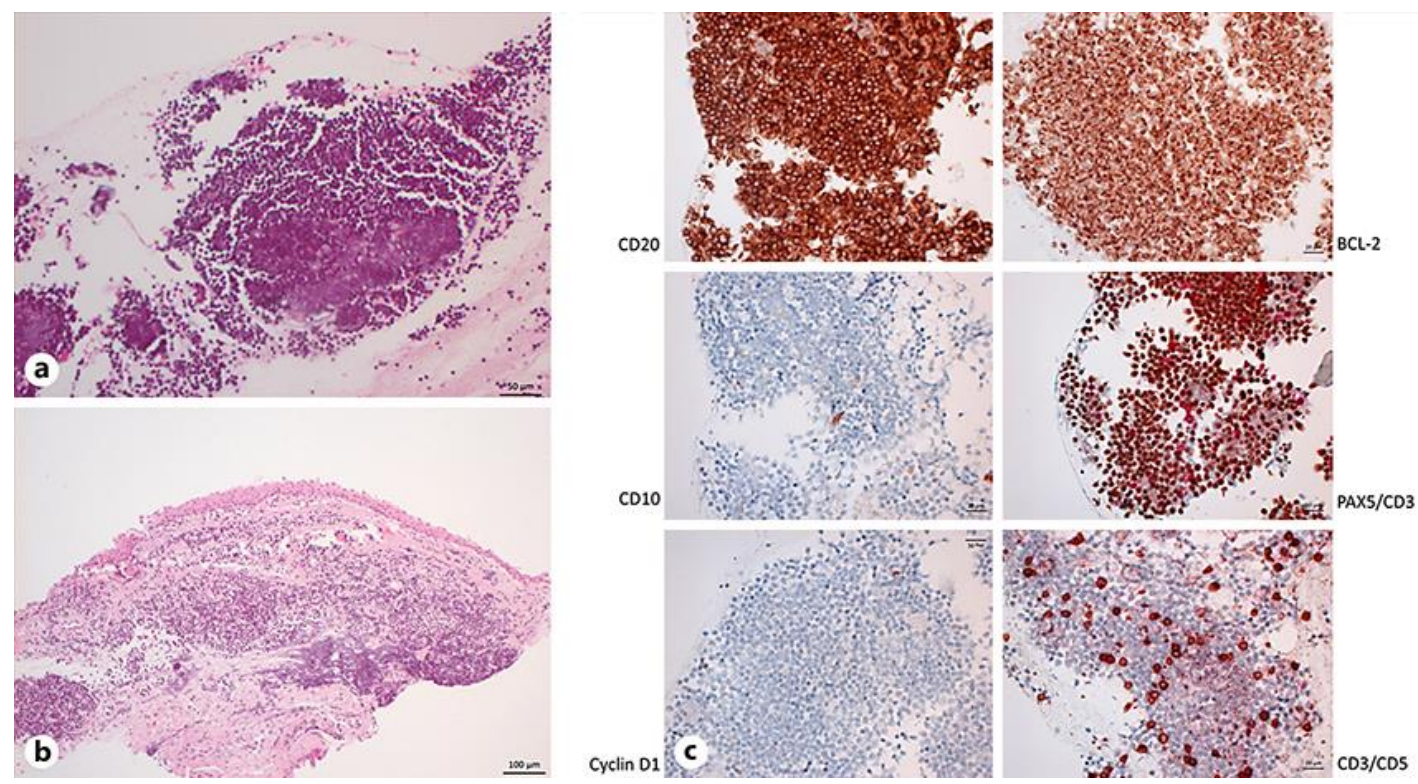

Fig. 2. a Cell block with haematoxylin and eosin stain of the choroidal biopsy demonstrates a monomorphous population of small lymphocytes. $\mathbf{b}$ The conjunctival biopsy demonstrates atypical lymphoid infiltrate in the substantia propria. c Immunohistochemical staining of the choroidal biopsy cell block shows a monomorphous population of CD20, bcl-2 positive, CD10, cyclin D1, CD23, and CD5 small lymphocytes.
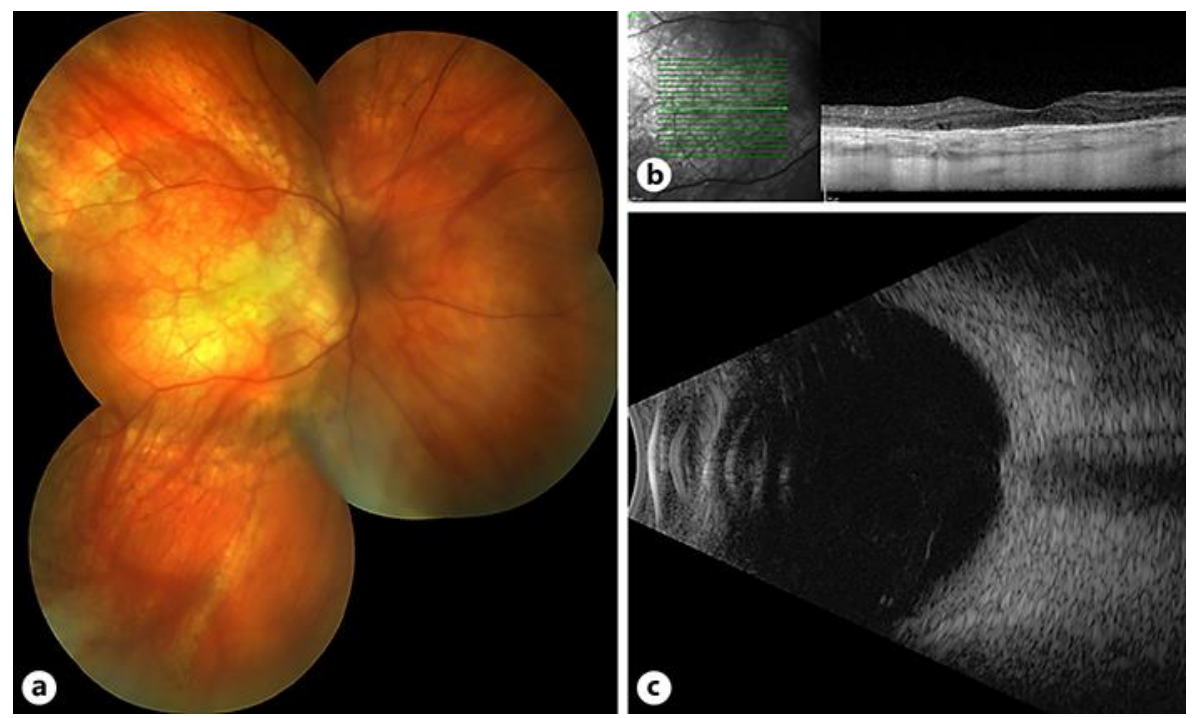

Fig. 3. a Colour fundus photograph of the right eye 3 months after commencing external beam radiotherapy reveals a residual area of pallor overlying the previous site of the choroidal lesion. $\mathbf{b}$ Enhanced depth imaging optical coherence tomography scan of the right macula shows resolution of the subretinal fluid and hyperreflective material and thinning of the choroid. c B-scan ultrasonography of the right eye reveals resolution of the choroidal thickening and extrascleral nodules. 